\title{
Doped Hole Transport Layer for Efficiency Enhancement in Planar Heterojunction Organolead
}

\section{Trihalide Perovskite Solar Cells}

\author{
Qi Wang, Cheng Bi and Jinsong Huang*
}

Department of Mechanical and Materials Engineering, University of Nebraska-Lincoln, Lincoln, Nebraska 68588, USA.

Keywords: organolead trihalide perovskite; photovoltaic; hole transport layer; doping.

Abstract: we demonstrated the efficiency of a solution-processed planar heterojunction organometallic trihalide perovskite solar cell can be increased to $17.5 \%$ through doping the hole transporting layer for reducing the resistivity. Doped Poly(triaryl amine) (PTAA) by 2,3,5,6-Tetrafluoro-7,7,8,8-tetracyanoquinodimethane (F4-TCNQ) reduced device series resistance by three-folds, increasing the device fill factor to $74 \%$, open circuit voltage to $1.09 \mathrm{~V}$ without sacrificing the short circuit current. This study reveals that the high resistivity of currently broadly applied polymer hole transport layer limits the device efficiency, and points a new direction to improve the device efficiency.

\section{Introduction}

In the past five years, organometallic trihalide perovskite (OTP) solar cells have been attracting tremendous interest because of their unique properties for photovoltaic applications, such as strong

\footnotetext{
*Correspond to: jhuang2@unl.edu
} 
1 absorption in the visible spectrum[1], long and balanced carrier diffusion length,[2-5] solution

2 processability and insensitivity to defect formation.[6, 7] Recently, a number of endeavors have been

3 directed to developing charge transport materials to further increase the efficiency of OTP photovoltaic

4 devices.[8-12] While a lot of new hole transport materials (HTMs) have been synthesized and applied

5 in the inverted structure OTP devices where HTMs were spun directly on top of the perovskite films, [8,

6 9, 11] most of these HTMs are difficult to be applied in regular structure planar heterojunction (PHJ)

7 OTP devices with the hole transport layer (HTL) underneath the perovskite layer. This is because the

8 general solvents used for the OTP, such as N,N-dimethylformamide (DMF) and dimethyl sulfoxide

9 (DMSO), have too good solubility and can wash off most commonly used small molecular HTMs, such as 2,2',7,7'-Tetrakis-(N,N-di-4-methoxyphenylamino)-9,9'-spirobifluorene (Spiro-OMeTAD). Therefore, although solution processed regular structure PHJ OTP solar cells have shown higher fill factor $(F F)$ of over than $80 \%$ and great promise to achieve higher power conversion efficiency (PCE), most of these PHJ OTP devices still adopted Poly(3,4-ethylenedioxythiophene) Polystyrene sulfonate (PEDOT:PSS) as HTL.[13-15] However, the low work function of PEDOT:PSS caused a relatively small open circuit voltage $\left(V_{O C}\right)$ of around $0.90 \mathrm{~V}$ in these PHJ devices, compared to the typical $V_{O C}$ of $>1.05 \mathrm{~V}$ in the inverted structure OTP devices.[14, 16-18] Recently, polymer HTMs have been studied because of its sustainability to DMF wash, for example, poly[ $N, N$ '-bis(4-butylphenyl)- $N, N \quad$ 'bis(phenyl)benzidine] (poly-TPD), was introduced into PHJ perovskite devices with device $V_{O C}$ greatly increased to 1.10 V.[19, 20] Meanwhile, we found that another polymer HTM, Poly(triaryl amine) (PTAA), also showed great capability to increase device $V_{O C}$ and survive the DMF wash. However, the PCE of these polymer HTLs based OTP devices was still around 15\% [19, 20]. Compared with the best 
1 reported efficiency of PEDOT:PSS based perovskite devices, the higher $V_{O C}$ of polymer HTL based

2 OPT devices was obtained at the expense of a smaller fill factor $(F F)$. $[13,14,16]$ We speculate that the

3 higher resistivity of these polymer HTMs than PEDOT:PSS may hinder charge extraction efficiency

4 which reduces $F F$ of these devices. Here, we report highly efficient solution-processed PHJ perovskite

5 solar cells using 2,3,5,6-Tetrafluoro-7,7,8,8-tetracyanoquinodimethane (F4-TCNQ) doped PTAA as the

6 HTL. F4-TCNQ doping was found to reduce the series resistance of the PTAA device by three times,

7 which improved the device PCE, especially when thick HTL was used.

\section{2. Experimental section}

\subsection{Device fabrication and characterization}

The methods of cleaning ITO glass, methylammonium iodide (MAI) synthesis can be found elsewhere.[13] The PTAA and F4-TCNQ were dissolved in toluene and mixed by desired F4-TCNQ/PTAA ratios ranging from $0.01 \mathrm{wt} \%$ to $10 \mathrm{wt} \%$. The mixture was stirred and heated at $70{ }^{\circ} \mathrm{C}$ overnight. Device with highest PCE was made by $10 \mathrm{mg} / \mathrm{ml}$ doped PTAA solution under a spin speed of 4000 RPM. For the device fabrication, small amount $(\sim 10$ wt $\%)$ of 4,4',4"-Tris[phenyl(m-tolyl)amino]triphenylamine (m-MTDATA) was mixed in the PTAA solution because the bare PTAA film was so smooth and non-wetting to DMF that $\mathrm{PbI}_{2}$ precursor was hardly spun on top of it. The perovskite films for device fabrication were made by interdiffusion method, in which $\mathrm{PbI}_{2}$ and MAI were sequentially spun on the substrates. The concentration of $\mathrm{PbI}_{2}$ was fixed at the $600 \mathrm{mg} / \mathrm{ml}$ and the concentration of MAI was varied from $50 \mathrm{mg} / \mathrm{ml}$ to $70 \mathrm{mg} / \mathrm{ml}$. The spun films were annealed at $100{ }^{\circ} \mathrm{C}$ on hotplate under the cover of a glass petri dish. $10 \mu \mathrm{L}-30 \mu \mathrm{L}$ of DMF 
1 solvent was added at the edge of the petri dish during annealing process. After that,

2 [6,6]-phenyl-C61-butyric acid methyl ester (PCBM) (dissolved in DCB, 2 wt\%) was spin coated on top

3 of the perovskite layer at $6000 \mathrm{RPM}$ for $35 \mathrm{~s}$. The films were annealed at $100{ }^{\circ} \mathrm{C}$ for $60 \mathrm{~min}$. Then 20

$4 \mathrm{~nm}$ thickness of $\mathrm{C}_{60}$ was thermally evaporated with a deposition rate of $0.5 \AA / \mathrm{s}$. The devices were

5 finished by the evaporation of $7 \mathrm{~nm}$ 2,9-dimethyl-4,7-diphenyl-1,10-phenanthroline (BCP) and $100 \mathrm{~nm}$

6 thick aluminum electrode.

7

8

9

Generally, the procedures for making PTAA films in the resistivity measurement were same with that for device fabrication. But thicker PTAA layer of $200 \mathrm{~nm}$ was used for increasing the signal-noise ratio. The schematic diagram of setup and the method of measuring resistivity can be found elsewhere.[21] The four probe measurement was conducted at atmosphere without encapsulation. Film thickness was measured by Bruker Dektak XTL step profiler. The photocurrents of the devices were measured under AM1.5G irradiation $\left(100 \mathrm{~mW} \mathrm{~cm}^{-2}\right)$ with a xenon-lamp-based solar simulator (Oriel 67005, 150W Solar Simulator). A Schott visible-colour glass-filtered (KG5 colour-filtered) Si diode (Hamamatsu S1133) was used to calibrate the light intensity before photocurrent measurement. Keithley 2400 Source-Meter was used for recording the I-V measurement.

\subsection{Fill factor Calculation}

The method of calculating FF was based on assumption that the maximum power output of the solar cell approximately equals to the power in the absence of series resistance minus the power lose of the series resistance.[22] 


$$
\mathrm{FF}=\mathrm{FF}^{\prime} *(1-\mathrm{r})
$$

2 where $\mathrm{FF}$ and $\mathrm{FF}^{\prime}$ are the fill factor of devices with and without series resistance, respectively. $\mathrm{r}$ and

3 ideal fill factor $\mathrm{FF}^{\prime}$ can be described by the following equations:[22]

4

5

6

7 where $R_{S}$ is the series resistance. $I_{M P}$ and $V_{M P}$ are the maximum-power-point current and voltage of

8 the device with negligible series resistance and infinitely large shunt resistance. $\mathrm{I}_{\mathrm{L}}$ is light generated

9 photocurrent, $\mathrm{I}_{0}$ is dark saturation current, $q$ is elemental charge, $\mathrm{n}$ is ideal factor, $k$ is Boltzmann constant, $\mathrm{T}$ is temperature. Here, we assumed dark saturation current equals to $1 * 10^{-16} \mathrm{~mA} / \mathrm{cm}^{2}$. Series resistance can be calculated based on resistivity measured by four probe method and the device geometry. Assuming $\mathrm{n}$ equals to $1, \mathrm{I}_{\mathrm{L}}$ equals to $24 \mathrm{~mA} / \mathrm{cm}^{2}$ based on the integrated sunlight spectrum of radiance, the device $F F s$ can be calculated by equation (1).

\section{Result and discussion}

Figure 1a shows the structure of the devices in this study. The perovskite films were made by interdiffusion method, where lead iodide $\left(\mathrm{PbI}_{2}\right)$ and methylammonium iodide (MAI) were sequentially spun onto the substrates, followed by a thermal annealing driven diffusion process.[14] It was discovered by us the surface of $\mathrm{MAPbI}_{3}$ can decompose during the thermal annealing process even though the annealing temperature was as low as $110{ }^{\circ} \mathrm{C}$. MAI was found to evaporate from the film surface, leaving $\mathrm{Pb}^{2+}$ rich surface, as revealed by the composition sensitive scanning probe microscopy 
1 technique.[24] Our density function theory calculation showed that the $\mathrm{Pb}^{2+}$ clusters cause deep

2 electron and hole traps on the perovskite surface.[24] Double-layer fullerenes were used as electron

3 transport layer, which can effectively passivate the charge traps on top of the perovskite film surface

4 generated by the surface decomposition during thermal annealing.[14, 16, 24] High concentration of

5 perovskite precursors was used in this study for stronger absorption. In addition, solvent annealing was

6 applied in this study for larger grain size.[16] Details about device fabrication and measurements could

7 be found in the experimental section. Figure $1 \mathrm{~b}$ shows the cross-section scanning electron microscopy

8 (SEM) of perovskite films on doped PTAA substrates. It is noted that the grain size of perovskite on

9 PTAA substrates is much larger than that on Pedot:PSS substrates.[14, 25] The non-wetting surface of

10 PTAA is beneficial for grain growth because of lower nucleus density and less dragging force.[26] The

11 larger grain size of several micrometers observed on the PTAA substrates are of importance in

12 suppressing grain boundary traps to obtain better device efficiency. Figure 1c and Figure 1d shows the

13 SEM images of perovskite on non-doped PTAA and $1 \mathrm{wt} \%$ F4-TCNQ doped PTAA film, respectively.

14 The morphology didn't exhibit noticeable difference before and after doping, excluding the influence

15 of dopant on perovskite morphology.

Figure 2a shows the current density-voltage $(\mathrm{J}-\mathrm{V})$ curves of devices with different F4-TCNQ doping concentration in the HTLs. The thickness of the HTL was kept unchanged by using a same solution concentration and spin rate. The changed doping level was found to have a significant impact on device performance, especially $F F$. As shown in the Figure $2 b$, the devices with 20 nm non-doped PTAA layer exhibited a low $F F$ of $65 \%$. After doping the PTAA layer by 1 wt $\%$ F4-TCNQ, the device 
1 FF was increased to $74 \%$. Further increasing doping level to $10 \mathrm{wt} \%$ deteriorated device PCE by

2 reducing FF to 64\%. The device with $1 \mathrm{wt} \%$ F4-TCNQ doped PTAA showed the highest efficiency

3 with a $J_{S C}$ of $21.6 \mathrm{~mA} / \mathrm{cm}^{2}$, a $V_{O C}$ of $1.09 \mathrm{~V}$, a $F F$ of $74 \%$ and PCE of $17.5 \%$ under 1 sun illumination.

4 Corresponding data can be found in Table I. Figure 2c-d show the external quantum efficiency (EQE),

5 steady photocurrent output and stabilized PCE of the device with 1 wt\% F4-TCNQ dopant in the HTL.

6 The calculated $J_{S C}$ of $21.0 \mathrm{~mA} / \mathrm{cm}^{2}$ from EQE is in good agreement with that from $\mathrm{J}-\mathrm{V}$ measurement.

7 And the steady photocurrent measured at the maximum power point $(0.85 \mathrm{~V})$ is the same with that

8 measured from J-V scanning, excluding the existence of obvious photocurrent hysteresis in our PHJ

9 devices. The stabilized efficiency is $17.5 \%$, confirming the accuracy of our device efficiency

characterization. The absence of photocurrent hysteresis can be explained by the large grain grown on

PTAA, and the application of double fullerene layer on the devices, which effectively reduce charge trap density at grain boundaries and film surface.[13, 27]

The $F F$ enhancement can be explained by the series resistance reduction of the PTAA HTL caused by F4-TCNQ doping. To verify this, the series resistance of devices with different F4-TCNQ doping concentrations was derived by the slope of J-V curves. As shown in Figure 3, doping the PTAA film by $1 \mathrm{wt} \%$ F4-TCNQ reduced the device series resistance from $9.07 \Omega * \mathrm{~cm}^{2}$ for non-doped PTAA to 6.07 $\Omega * \mathrm{~cm}^{2}$. Further increasing F4-TCNQ doping level to $10 \mathrm{wt} \%$ increased the device series resistance to 9.77 $\Omega * \mathrm{~cm}^{2}$, most likely caused by the increased aggregation of F4-TCNQ.[28-31] The series resistance derived from the $\mathrm{J}-\mathrm{V}$ curve slope can be an indirect evidence for the doping effect because it represents the series resistance of the whole device rather than the PTAA films. To confirm the doping 
1 effect of F4-TCNQ, we further conducted four-probe measurements on the PTAA films with different

2 doping levels. Details about four probe measurement can be found in the experimental section and our

3 previous publication.[21] It should be noted that $200 \mathrm{~nm}$ PTAA film, which is much thicker than the

4 PTAA layer in devices, was used to reduce noise in the four-probe measurement. To estimate the series

5 resistance of the PTAA in real devices, the series resistance got from four-probe method was divided by

6 a factor by assuming the series resistance has a liner relationship with the PTAA thickness. As shown in

7 Figure 3, the resistivity variation measured by four-probe method follows the same trend with that

8 derived from $\mathrm{J}-\mathrm{V}$ curves, confirming F4-TCNQ doping can change the PTAA resistivity. It is noted that

9 the resistance measured by four-probe method is slightly larger than the resistance measured by $\mathrm{J}-\mathrm{V}$

10 slope. One possible reason is four-probe method measures the resistivity of film in lateral direction and

11 the resistivity of PTAA film in the lateral and vertical directions can be different. Another possible

12 reason is the electric field in $\mathrm{J}-\mathrm{V}$ measurement is much larger than that in the four-probe measurement.

13 In addition, the thickness of PTAA layer in devices was thin and may barely cover the whole substrate.

Now that we have demonstrated the doping effect of F4-TCNQ in changing the resistivity of the

PTAA layer, the influence of resistance on device efficiency needs to be clarified. Here, we applied the equivalent circuit and simulation method in inorganic solar cell to calculate $F F$ s. Details about the calculation can be found in the experimental section. The two curves in Figure $2 \mathrm{~b}$ are the device FFs derived and $\mathrm{J}-\mathrm{V}$ measured. The calculated FF variation is in good agreement with the measured data, indicating series resistance variation is the primary reason for the $F F$ change in our devices. The calculation also implies the importance of reducing series resistance for further device PCE 
5

enhancement. The up limit of $F F$ was calculated from equation (4) in the experimental section to be $89 \%$, which provides a large potential for efficiency enhancement and great promise to boost PCE exceeding $20 \%$. Since F4-TCNQ can only change the resistivity by several times, better dopants are expected in the future study to further reducing the resistivity and increasing $F F$.

Another merit for F4-TCNQ doping is that the reduced HTL resistance should make the device efficiency less dependent on HTL thickness. To demonstrate it, we compared the devices with doped or non-doped PTAA HTLs with different HTL thicknesses from $20 \mathrm{~nm}$ to $70 \mathrm{~nm}$. Figure 4a-b show the $\mathrm{J}-\mathrm{V}$ curves of devices with and without F4-TCNQ doping, respectively. Without doping, the device J-V curve gradually showed s-kink when the HTL thickness increased to $70 \mathrm{~nm}$. On the other hand, the devices with 1 wt\% F4-TCNQ doped PTAA HTLs have PCE almost insensitive to PTAA film thickness.

Figure $4 \mathrm{c}$ summarizes the series resistance of the devices with non-doped and doped PTAA of different thicknesses. For the devices containing $20 \mathrm{~nm}$ HTL, 1 wt\% F4-TCNQ doping reduced the series resistance from $8.8 \Omega * \mathrm{~cm} 2$ to $6.2 \Omega * \mathrm{~cm} 2$. Lower series resistance was observed in the doped device, especially when the HTL is thick. The resistivity of the device with $70 \mathrm{~nm}$ HTL was reduced three times from $26.6 \Omega * \mathrm{~cm} 2$ to $9.33 \Omega * \mathrm{~cm} 2$ after doping with $1 \mathrm{wt} \% \mathrm{~F} 4-\mathrm{TCNQ}$. The insensitivity of device PCE on HTL thickness is benefit for device reproducibility and especially attractive for large-area device fabrication.

\section{Conclusion}

In conclusion, we reported the increased device FF and enhanced device PCE of OTP solar cells 
1 using a doped polymer hole transport layer. By optimizing doping level, a high device PCE of $17.5 \%$

2 was achieved. This study emphasized the advantages of doping hole transport layer on the PCE of OTP

3 solar cells and pointed out a direction of further increasing the efficiency of OTP PHJ solar cells to

4 above $20 \%$ by exploring better dopants.

\section{Acknowledgements:}

7 This work was partially supported by the SunShot Program of the US Department of Energy (Energy

8 Efficiency and Renewable Energy) under award number DE-EE0006709 (Q. W., experiment, analysis)

9 and Nebraska Public Power District through the Nebraska Center for Energy Sciences Research (C. B

10 for SEM and experiment direction).

\section{References:}

[1] M.M. Lee, J. Teuscher, T. Miyasaka, T.N. Murakami, H.J. Snaith, Efficient hybrid solar cells based on meso-superstructured organometal halide perovskites, Science, 338 (2012) 643-647.

[2] S.D. Stranks, G.E. Eperon, G. Grancini, C. Menelaou, M.J. Alcocer, T. Leijtens, L.M. Herz, A.

Petrozza, H.J. Snaith, Electron-hole diffusion lengths exceeding 1 micrometer in an organometal trihalide perovskite absorber, Science, 342 (2013) 341-344.

[3] Q. Dong, Y. Fang, Y. Shao, P. Mulligan, J. Qiu, L. Cao, J. Huang, Electron-hole diffusion lengths $>175 \mu \mathrm{m}$ in solution grown CH3NH3PbI3 single crystals, Science, (2015 DOI: 10.1126/science.aaa5760).

[4] G. Xing, N. Mathews, S. Sun, S.S. Lim, Y.M. Lam, M. Grätzel, S. Mhaisalkar, T.C. Sum, 
1

2 [5] D. Shi, V. Adinolfi, R. Comin, M. Yuan, E. Alarousu, A. Buin, Y. Chen, S. Hoogland, A.

3 Rothenberger, K. Katsiev, Low trap-state density and long carrier diffusion in organolead trihalide

4 perovskite single crystals, Science, 347 (2015) 519-522.

5 [6] W.J. Yin, T. Shi, Y. Yan, Unique Properties of Halide Perovskites as Possible Origins of the Superior

6 Solar Cell Performance, Adv. Mater., 26 (2014) 4653.

7 [7] W.-J. Yin, T. Shi, Y. Yan, Unusual defect physics in CH3NH3PbI3 perovskite solar cell absorber, $8 \quad$ Appl. Phys. Lett., 104 (2014) 063903.

9 [8] N.J. Jeon, J. Lee, J.H. Noh, M.K. Nazeeruddin, M. Grätzel, S.I. Seok, Efficient inorganic-organic 10

342 (2013) 344-347. hybrid perovskite solar cells based on pyrene arylamine derivatives as hole-transporting materials, J. Am. Chem. Soc., 135 (2013) 19087-19090.

[9] P. Qin, S. Paek, M.I. Dar, N. Pellet, J. Ko, M. Grätzel, M.K. Nazeeruddin, Perovskite Solar Cells with 12.8\% Efficiency by Using Conjugated Quinolizino Acridine Based Hole Transporting Material, J. Am. Chem. Soc., 136 (2014) 8516.

[10] J.H. Kim, P.W. Liang, S.T. Williams, N. Cho, C.C. Chueh, M.S. Glaz, D.S. Ginger, A.K.Y. Jen, High-Performance and Environmentally Stable Planar Heterojunction Perovskite Solar Cells Based on a Solution-Processed Copper-Doped Nickel Oxide Hole-Transporting Layer, Adv. Mater., 27 (2014) 695.

[11] J. Liu, Y. Wu, C. Qin, X. Yang, T. Yasuda, A. Islam, K. Zhang, W. Peng, W. Chen, L. Han, A dopant-free hole-transporting material for efficient and stable perovskite solar cells, Energ. Environ. Sci., 7 (2014) 2963-2967. 
1

2 3

4

[12] J.A. Christians, R.C. Fung, P.V. Kamat, An inorganic hole conductor for organo-lead halide perovskite solar cells. Improved hole conductivity with copper iodide, J. Am. Chem. Soc., 136 (2013) 758-764.

[13] Q. Wang, Y. Shao, Q. Dong, Z. Xiao, Y. Yuan, J. Huang, Large fill-factor bilayer iodine perovskite solar cells fabricated by a low-temperature solution-process, Energ. Environ. Sci., 7 (2014) 2359-2365.

[14] Z. Xiao, C. Bi, Y. Shao, Q. Dong, Q. Wang, Y. Yuan, C. Wang, Y. Gao, J. Huang, Efficient, High Yield Perovskite Photovoltaic Devices Grown by Interdiffusion of Solution-Processed Precursor Stacking Layers, Energ. Environ. Sci., 7 (2014) 2619-2623.

[15] W. Nie, H. Tsai, R. Asadpour, J.-C. Blancon, A.J. Neukirch, G. Gupta, J.J. Crochet, M. Chhowalla, S. Tretiak, M.A. Alam, H.-L. Wang, A.D. Mohite, High-efficiency solution-processed perovskite solar cells with millimeter-scale grains, Science, 347 (2015) 522-525.

[16] Z. Xiao, Q. Dong, C. Bi, Y. Shao, Y. Yuan, J. Huang, Solvent Annealing of Perovskite-Induced Crystal Growth for Photovoltaic-Device Efficiency Enhancement, Adv. Mater., 26 (2014) 6503-6509.

[17] J.M. Ball, M.M. Lee, A. Hey, H.J. Snaith, Low-temperature processed meso-superstructured to thin-film perovskite solar cells, Energ. Environ. Sci., 6 (2013) 1739-1743.

[18] J.-H. Im, I.-H. Jang, N. Pellet, M. Grätzel, N.-G. Park, Growth of CH3NH3PbI3 cuboids with controlled size for high-efficiency perovskite solar cells, Nat. Nanotechnol., 9 (2014) 927-932.

[19] D. Zhao, M. Sexton, H.Y. Park, G. Baure, J.C. Nino, F. So, High-Efficiency Solution-Processed Planar Perovskite Solar Cells with a Polymer Hole Transport Layer, Adv. Energy Mater., DOI: 10.1002/aenm.201401855 (2014).

[20] O. Malinkiewicz, C. Roldán-Carmona, A. Soriano, E. Bandiello, L. Camacho, M.K. Nazeeruddin, 
H.J. Bolink, Metal-Oxide-Free Methylammonium Lead Iodide Perovskite-Based Solar Cells: the Influence of Organic Charge Transport Layers, Adv. Energy Mater., DOI: 10.1002/aenm.201400345 (2014).

[21] Q. Wang, Y. Shao, H. Xie, L. Lyu, X. Liu, Y. Gao, J. Huang, Qualifying composition dependent p and n self-doping in CH3NH3PbI3, Appl. Phys. Lett., 105 (2014) 163508.

[22] N. Koide, A. Islam, Y. Chiba, L. Han, Improvement of efficiency of dye-sensitized solar cells based on analysis of equivalent circuit, J. Photoch. Photobio. A: Chem., 182 (2006) 296-305.

[23] K. Vandewal, K. Tvingstedt, A. Gadisa, O. Inganäs, J.V. Manca, On the origin of the open-circuit voltage of polymer-fullerene solar cells, Nat. Mater., 8 (2009) 904-909.

[24] R. Dong, Y. Fang, J. Chae, J. Dai, Z. Xiao, Q. Dong, Y. Yuan, A. Centrone, X.C. Zeng, J. Huang, High-Gain and Low-Driving-Voltage Photodetectors Based on Organolead Triiodide Perovskites, Adv. Mater., DOI: 10.1002/adma.201405116 (2015).

[25] C. Bi, Y. Shao, Y. Yuan, Z. Xiao, C. Wang, Y. Gao, J. Huang, Understanding the formation and evolution of interdiffusion grown organolead halide perovskite thin films by thermal annealing, $\mathrm{J}$. Mater. Chem. A, 2 (2014) 18508-18514.

[26] C. Bi, Q. Dong, Y. Shao, Q. Wang, Y. Yuan, Z. Xiao, J. Huang, Nonwetting Surface Driven High Aspect Ratio Crystalline Grain Growth for Efficient Hybrid Perovskite Solar Cells, Nat. Commun., (under revision) (2015).

[27] Y. Shao, Z. Xiao, C. Bi, Y. Yuan, J. Huang, Origin and elimination of photocurrent hysteresis by fullerene passivation in CH3NH3PbI3 planar heterojunction solar cells, Nat. Commun., 5 (2014) 5784.

[28] D.T. Duong, H. Phan, D. Hanifi, P.S. Jo, T.Q. Nguyen, A. Salleo, Direct Observation of Doping 
1 Sites in Temperature-Controlled, p-Doped P3HT Thin Films by Conducting Atomic Force Microscopy,

2 Adv. Mater., 26 (2014) 6069-6073.

3 [29] F. Deschler, D. Riedel, A. Deák, B. Ecker, E. von Hauff, E. Da Como, Imaging of morphological

4 changes and phase segregation in doped polymeric semiconductors, Synth. Metals, 199 (2015)

$5 \quad 381-387$.

6 [30] X. Han, Z. Wu, B. Sun, Enhanced performance of inverted organic solar cell by a solution-based

7 fluorinated acceptor doped P3HT: PCBM layer, Org. Electron., 14 (2013) 1116-1121.

8 [31] P. Pingel, L. Zhu, K.S. Park, J.r.-O. Vogel, S. Janietz, E.-G. Kim, J.r.P. Rabe, J.-L. Brédas, N. Koch,

9 Charge-transfer localization in molecularly doped thiophene-based donor polymers, J. Phy. Chem. Lett., $10 \quad 1(2010) 2037-2041$. 
1 Table I Perovskite device performance with different F4-TCNQ doping levels in PTAA layers. RS is device series

2 resistances derived from I-V curves and $\mathrm{R}_{\mathrm{S}}^{\prime}$ is series resistances of PTAA films derived from four-probe method.

\begin{tabular}{ccccccc}
\hline Doping ratio & $\begin{array}{c}\mathrm{J}_{\mathrm{SC}} \\
(\mathrm{mA} / \mathrm{cm} 2)\end{array}$ & $\mathrm{V}_{\mathrm{OC}}(\mathrm{V})$ & $\mathrm{FF}(\%)$ & $\mathrm{PCE}(\%)$ & $\mathrm{R}_{\mathrm{S}}\left(\Omega^{*} \mathrm{~cm} 2\right)$ & $\mathrm{R}_{\mathrm{S}}^{\prime}\left(\Omega^{*} \mathrm{~cm} 2\right)$ \\
\hline $0 \mathrm{wt} \%$ & 21.6 & 1.05 & 65.7 & 14.8 & 9.07 & 12.9 \\
$0.01 \mathrm{wt} \%$ & 21.0 & 1.05 & 65.8 & 14.5 & 7.57 & 12.1 \\
$0.1 \mathrm{wt} \%$ & 21.5 & 1.05 & 65.5 & 14.8 & 7.72 & 10.6 \\
$1 \mathrm{wt} \%$ & 21.6 & 1.09 & 74.0 & 17.5 & 6.07 & 8.4 \\
$2 \mathrm{wt} \%$ & 21.0 & 1.09 & 68.2 & 15.6 & 8.56 & 8.2 \\
$10 \mathrm{wt} \%$ & 21.2 & 1.09 & 64.1 & 14.8 & 9.77 & 11.4 \\
\hline
\end{tabular}

3

4 


\section{Figure Captions:}

$3 \quad$ Figure 1 (a) Schematic structure of the devices in this study. The thickness of each layer was not in

4 scale with the real thickness for clarity. (b) Cross-section scanning electron microscopy picture of 5 perovskite on 1 wt\% F4-TCNQ doped PTAA layer. SEM images of perovskite films on non-doped PTAA

(c) and 1 wt\% F4-TCNQ doped PTAA (d).

8 Figure 2 (a) The photocurrents of the devices under AM 1.5 simulated illumination with different doping levels of PTAA layer. (b) Calculated and experimental fill factors of devices with different doping levels of HTL. External quantum efficiency (c) and steady maximum power output measurement (d) of the best device.

Figure 3 Series resistance of PTAA films with different F4-TCNQ doping levels. The series resistance was measured by four probe method and slope of device I-V curve.

Figure 4 Photocurrents of the devices with doped (a) or non-doped (b) HTL under AM 1.5 simulated illumination. The HTL thickness was varied from 20 to $70 \mathrm{~nm}$. (c) Corresponding series resistance of the doped or non-doped HTL films derived from the slope of I-V curves. 


\section{Figure 1}

(a)

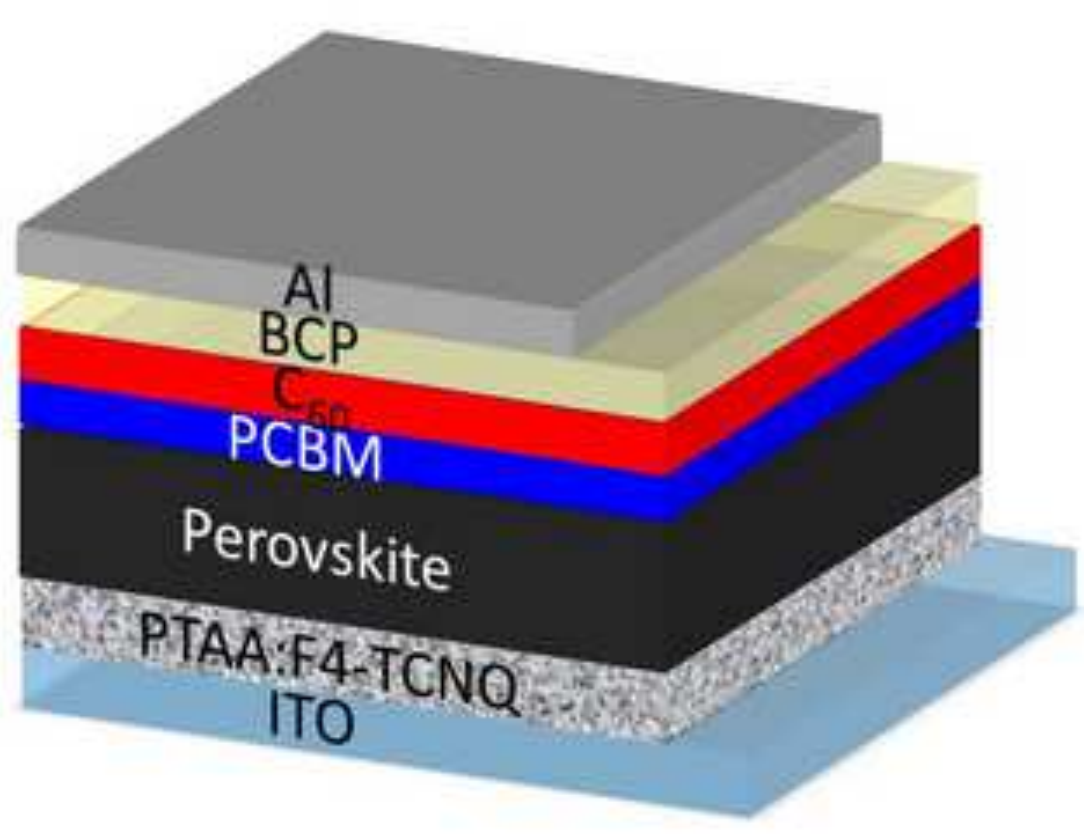

(c)

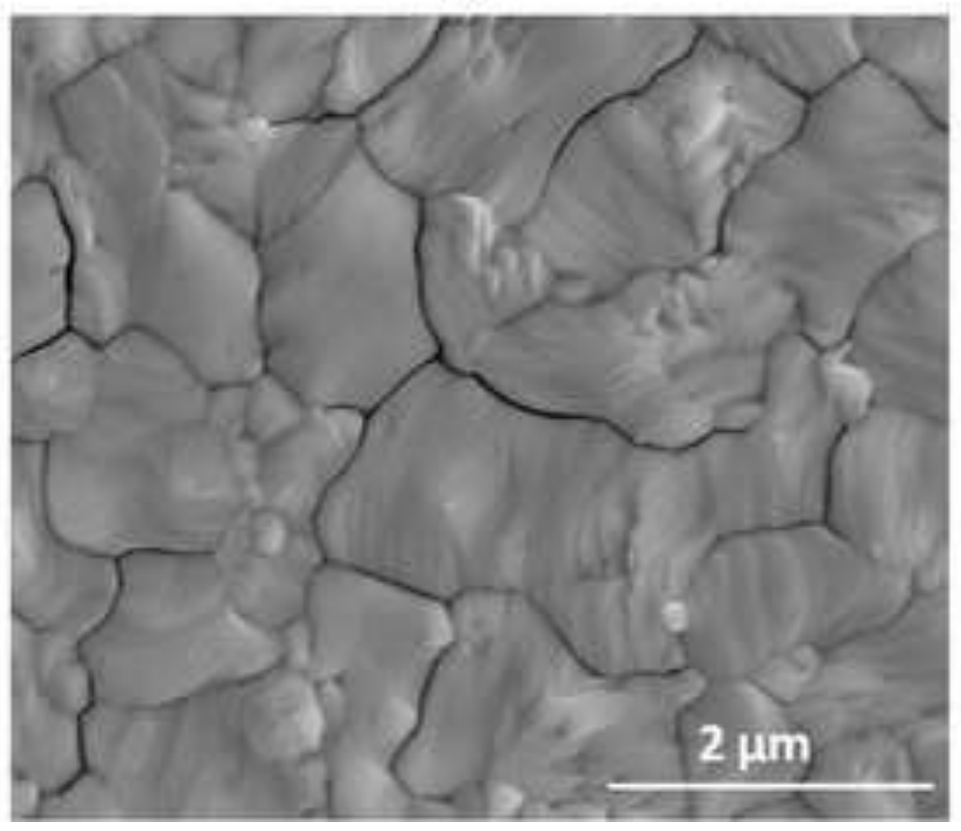

(b)

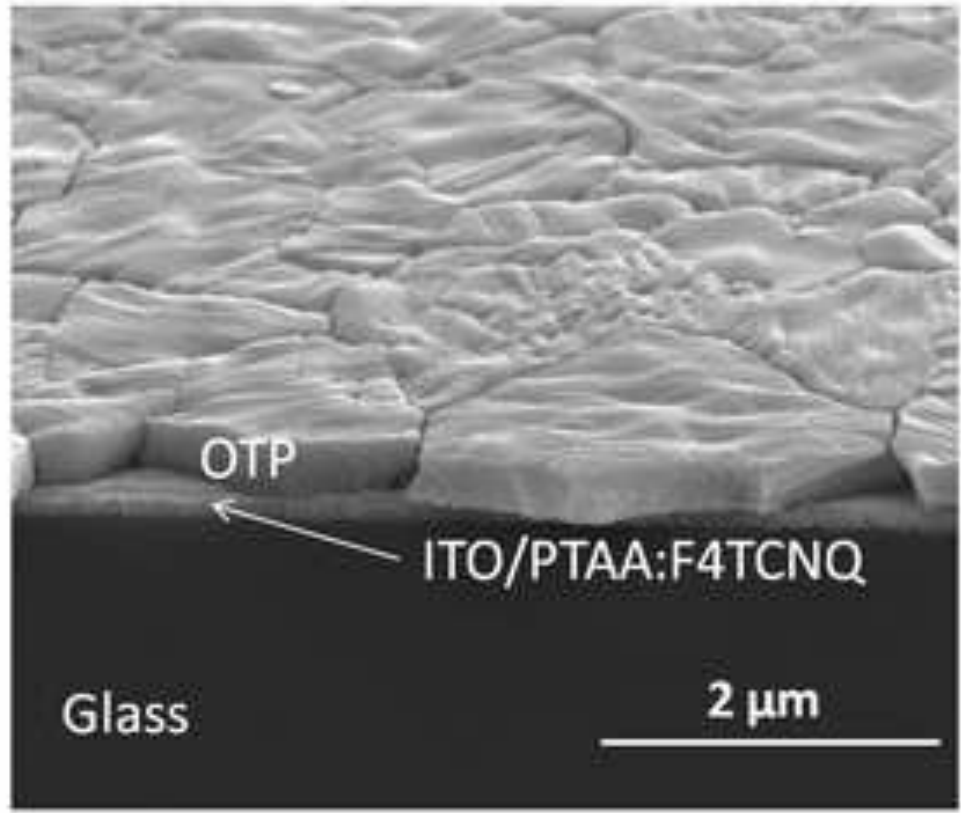

(b)

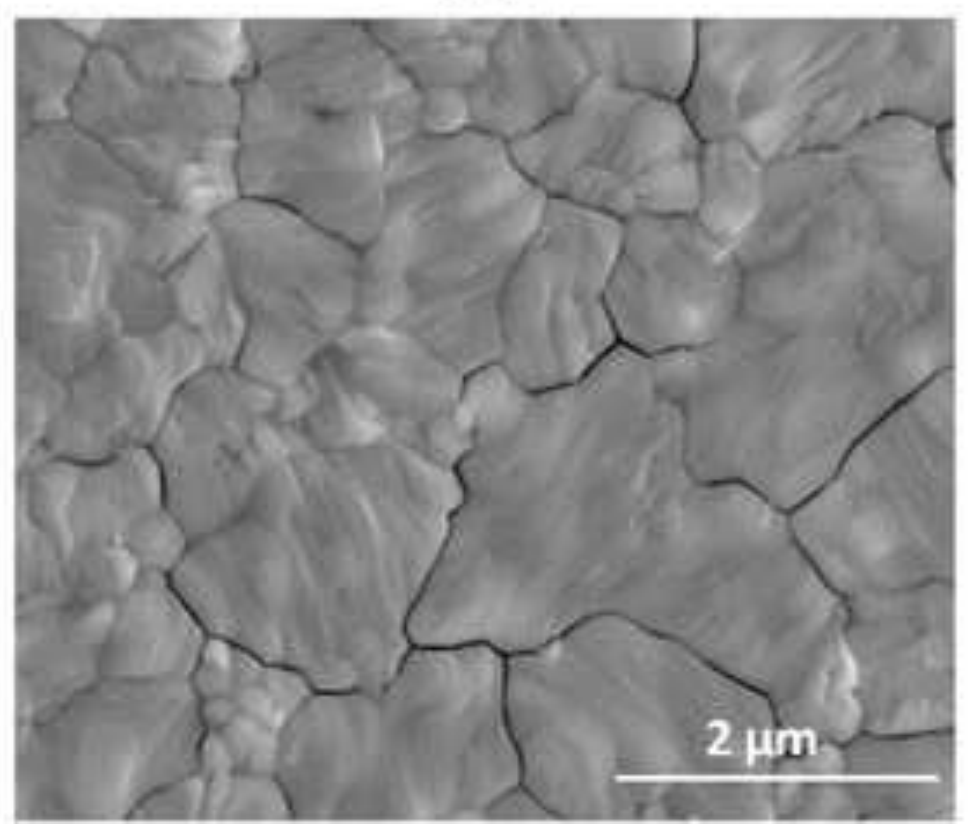


(a)

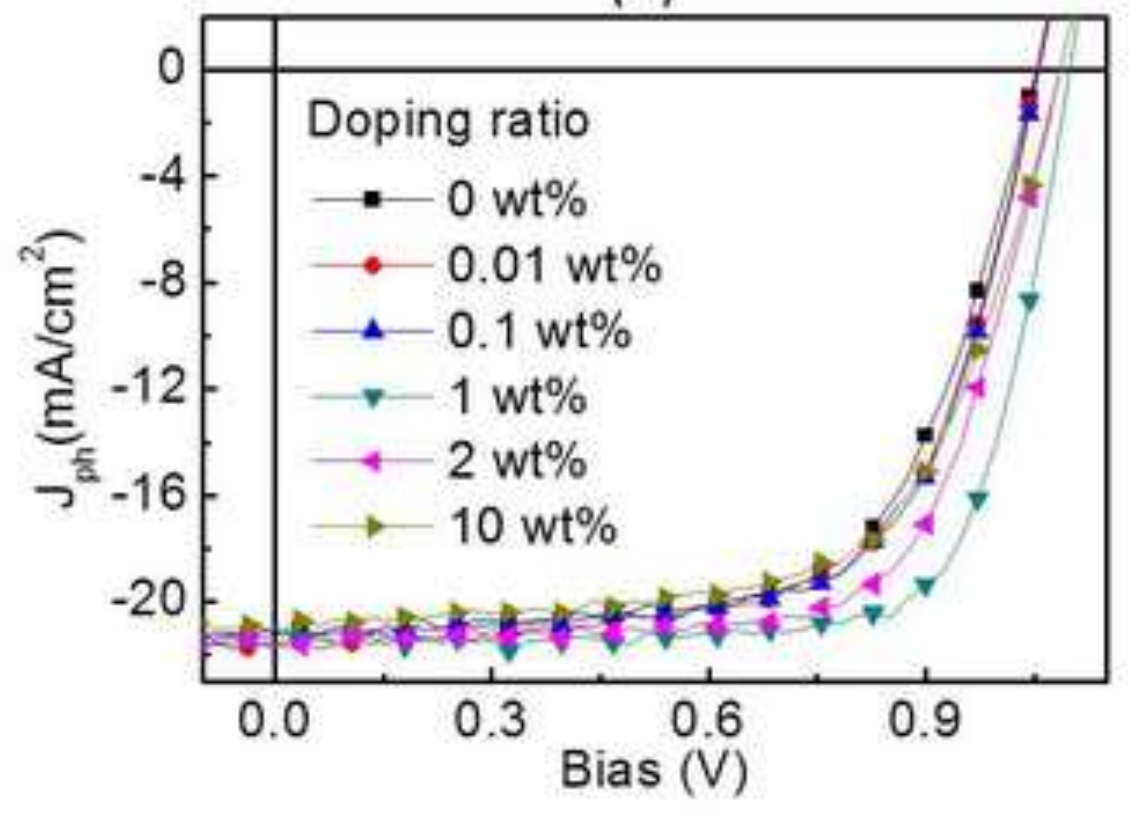

(c)

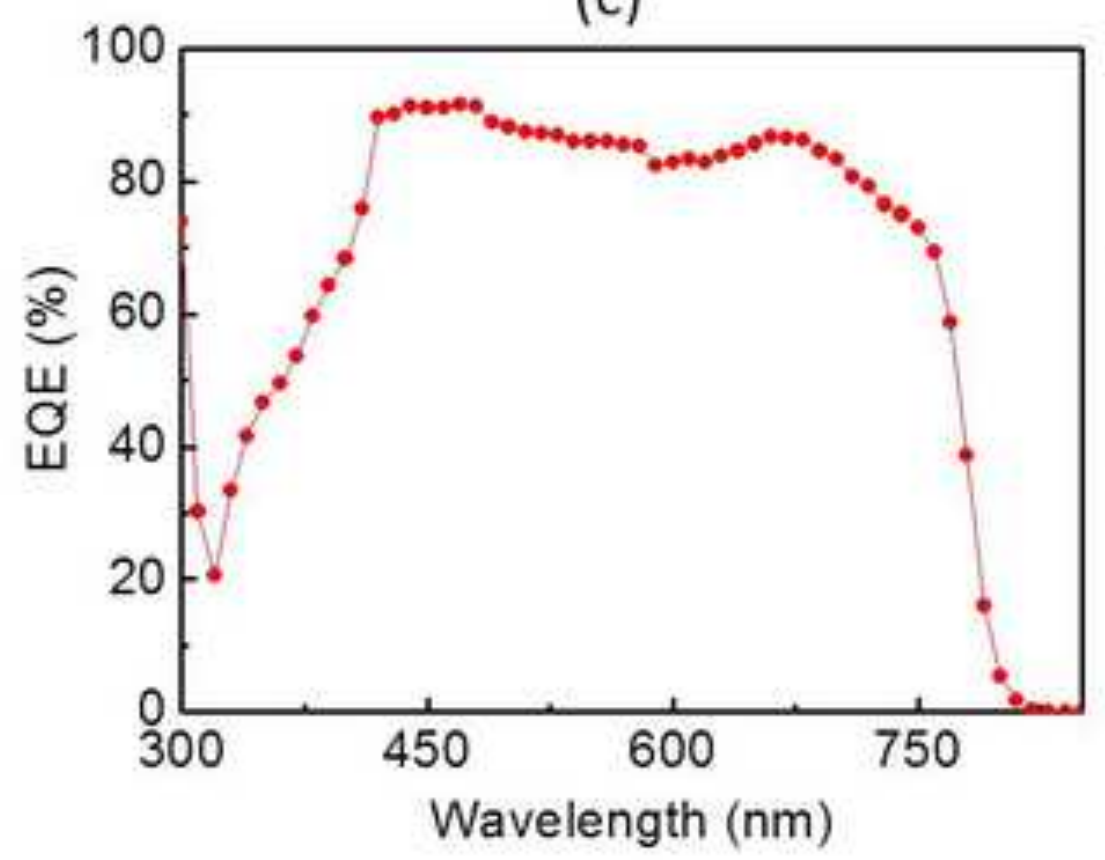

(b)

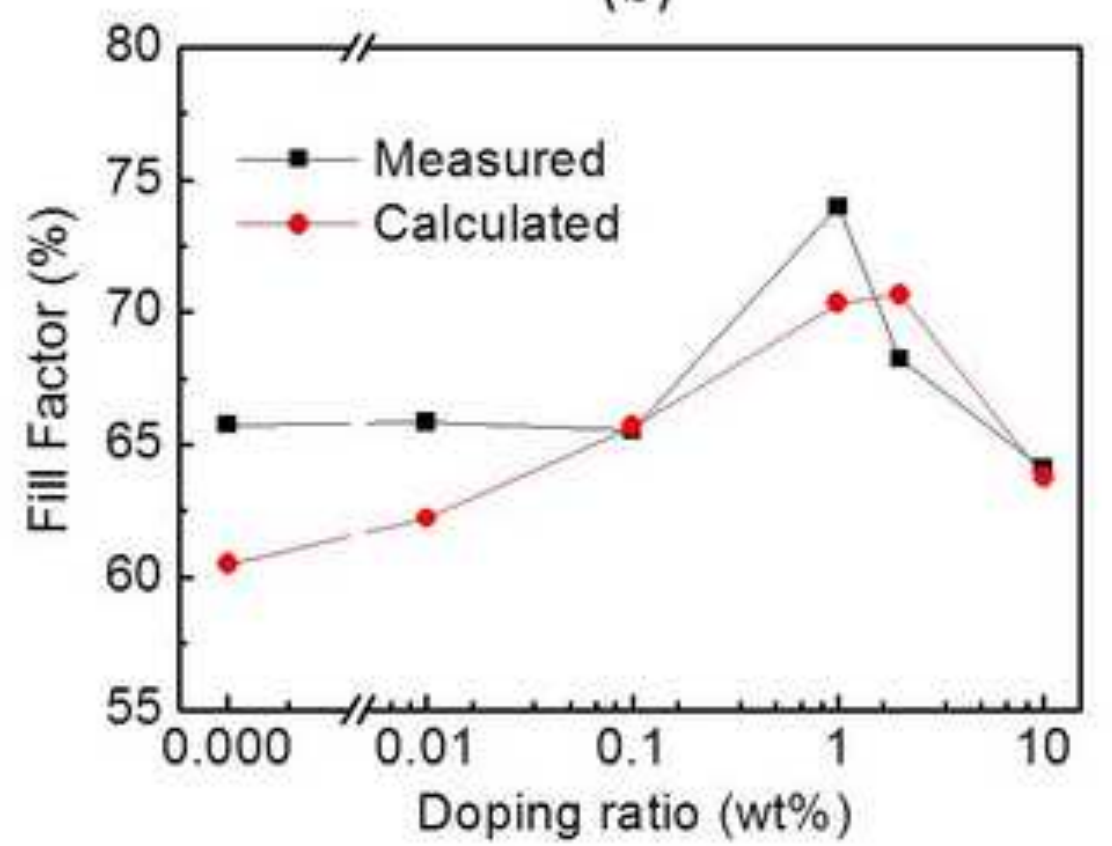

(d)

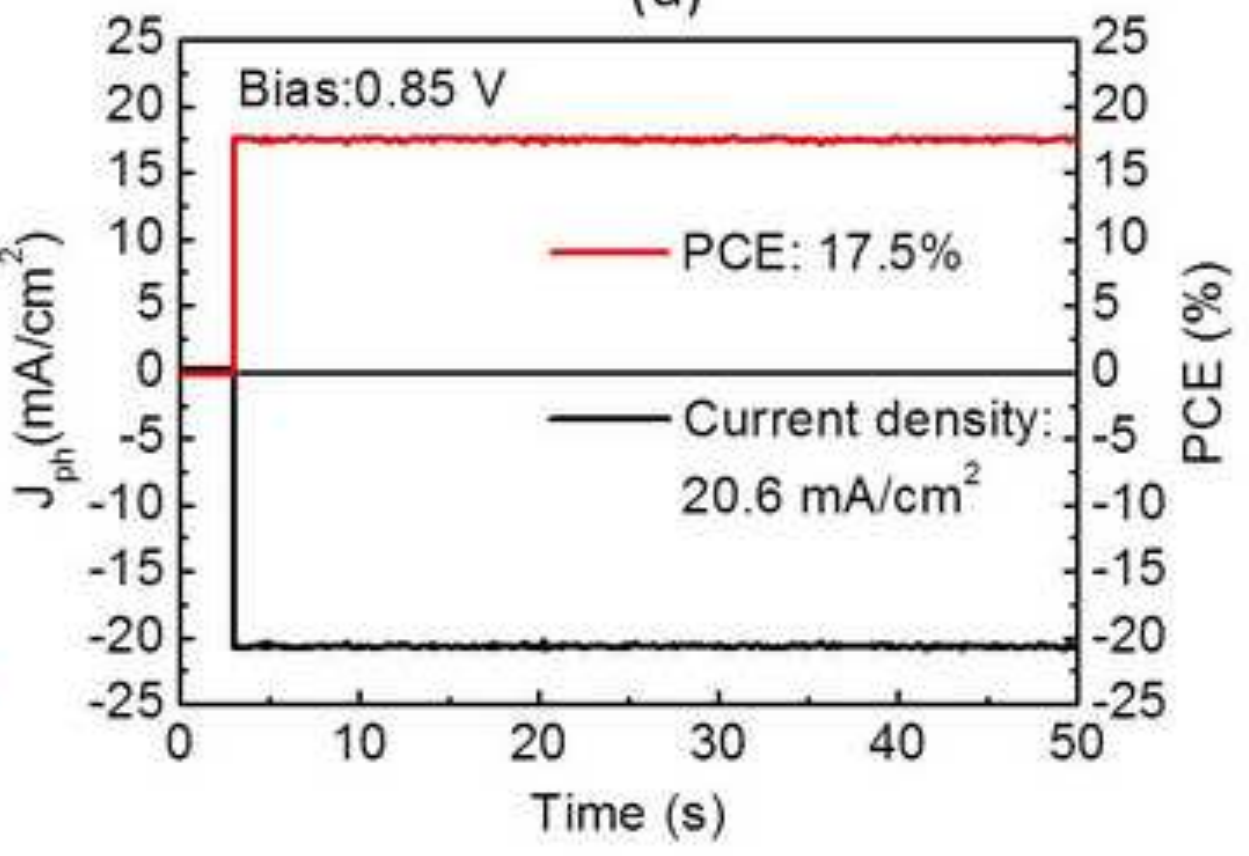




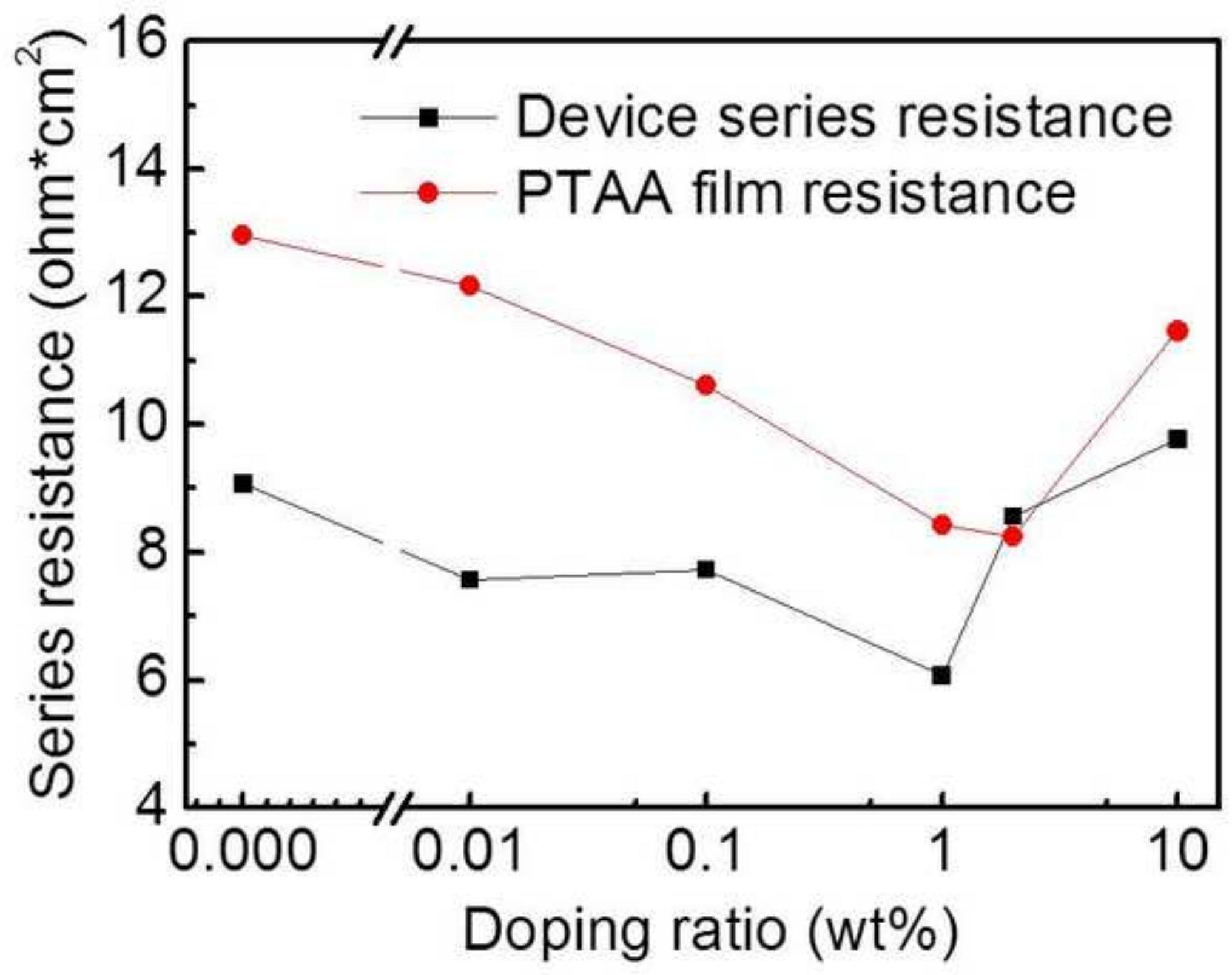


(a)

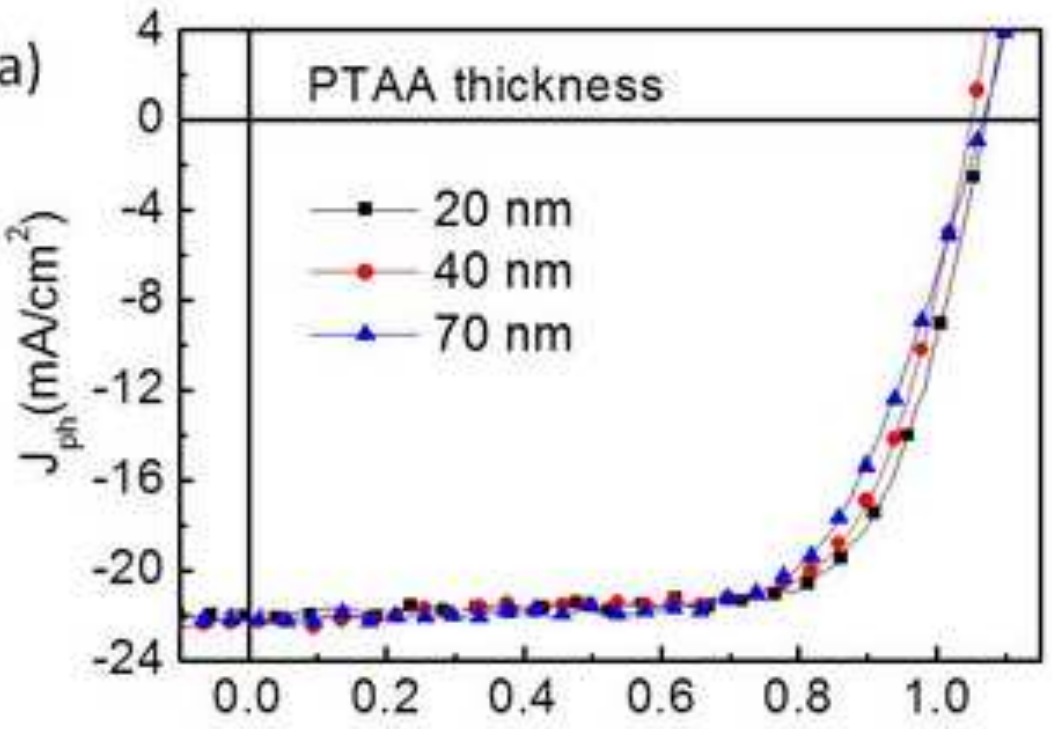

(b)

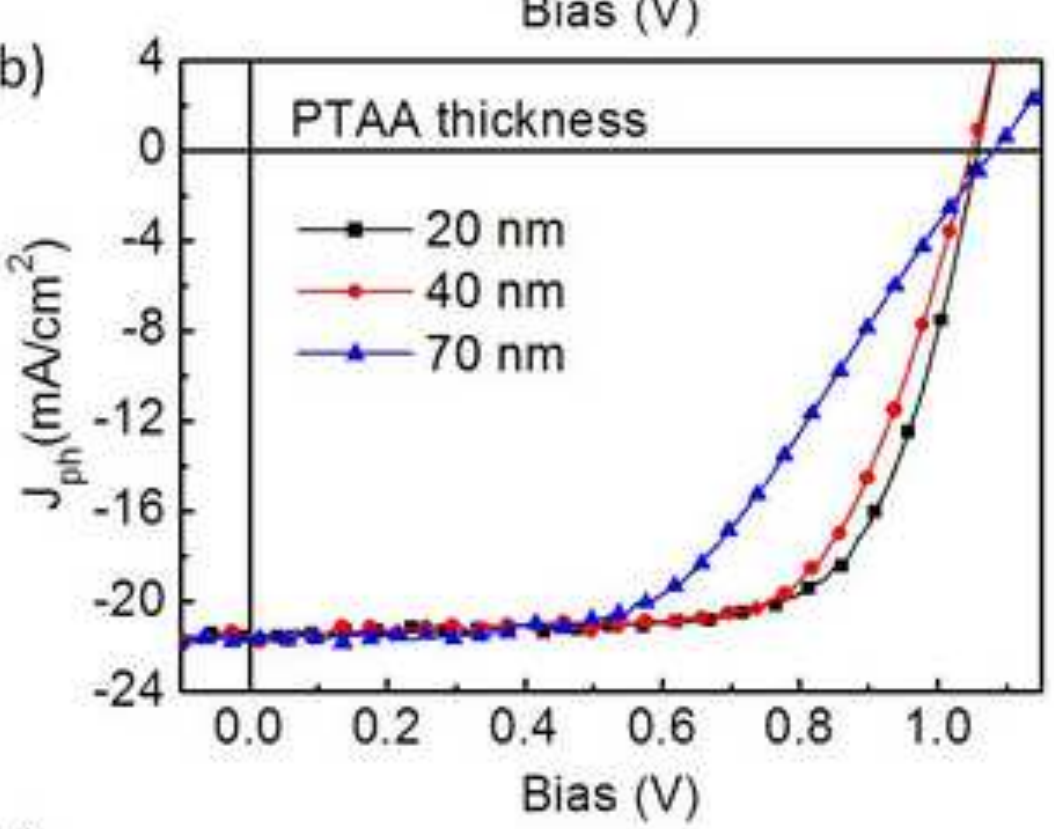

(c)

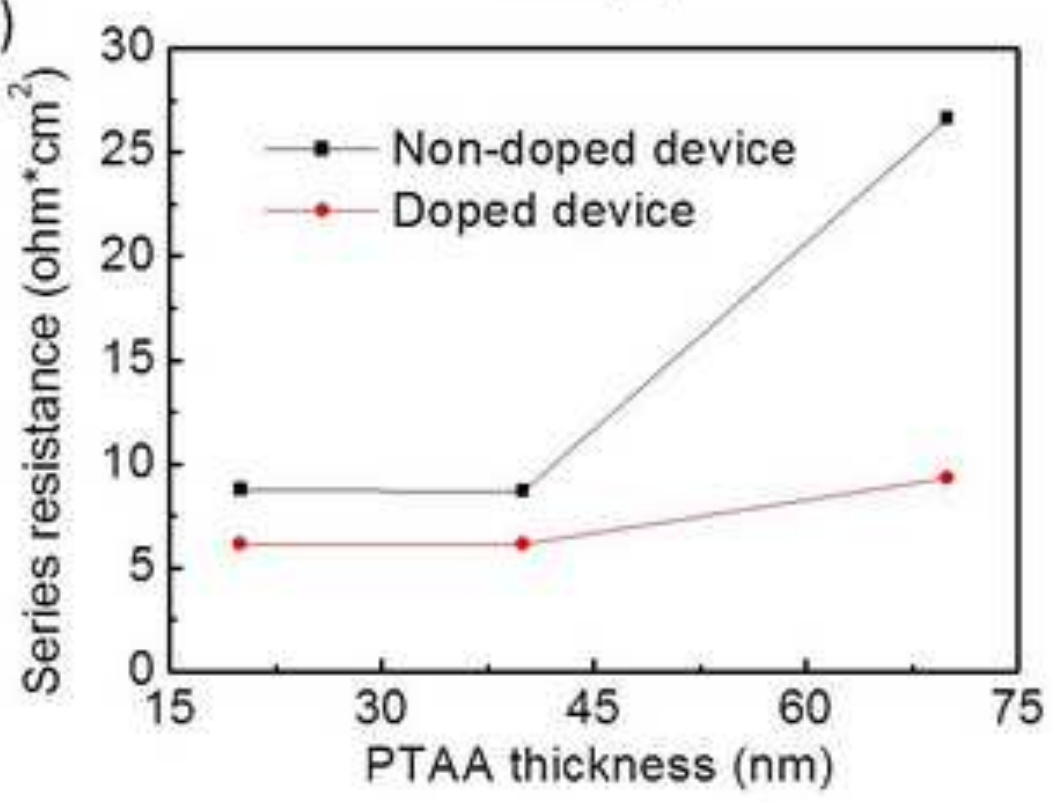



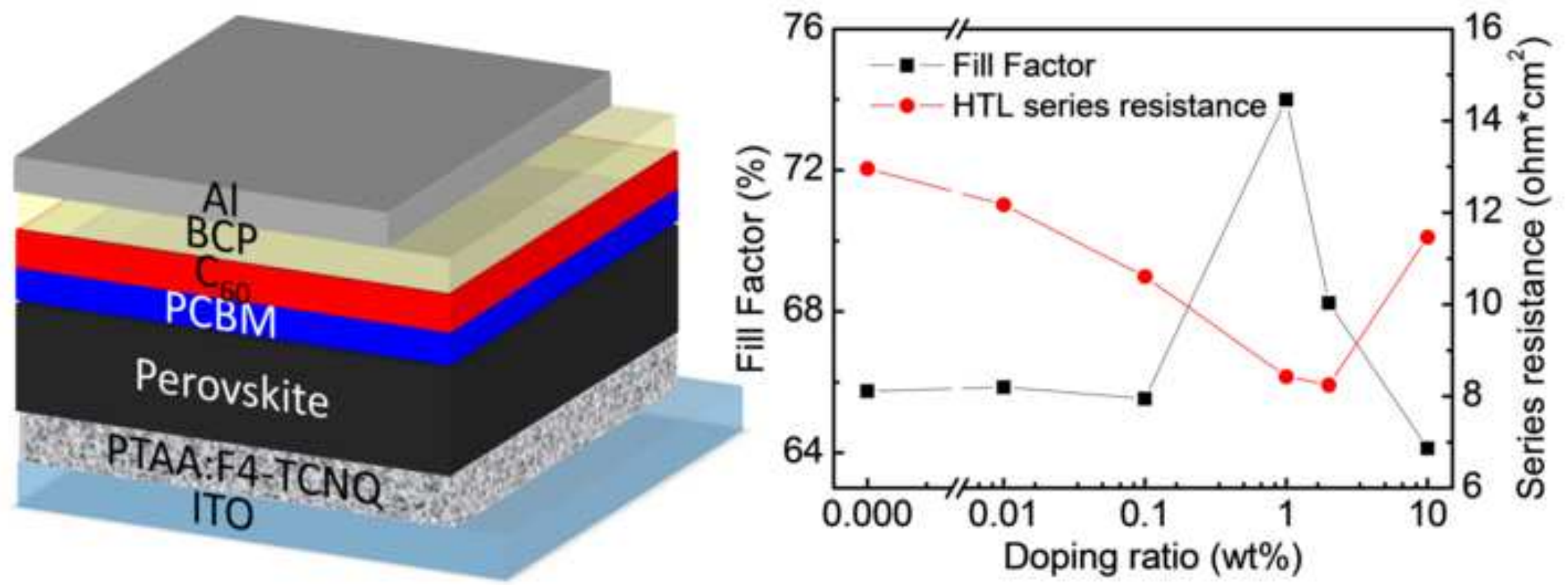